\title{
El principio de formas de implicación y motivación del diseño universal para el aprendizaje con una mirada a la política curricular de Costa Rica
}

Quirós Acuña, Maybel; Alberto Segura, Mario

El principio de formas de implicación y motivación del diseño universal para el aprendizaje con una mirada a la politica curricular de Costa Rica

Revista Educación, vol. 44, núm. 2, 2020

Universidad de Costa Rica, Costa Rica

Disponible en: http://www.redalyc.org/articulo.oa?id=44062184034

DOI: https://doi.org/10.15517/revedu.v44i2.40360

Esta obra está bajo una Licencia Creative Commons Atribución-NoComercial-SinDerivar 3.0 Internacional. 


\title{
El principio de formas de implicación y motivación del diseño universal para el aprendizaje con una mirada a la política curricular de Costa Rica
}

\author{
The Principle of Engagement and Motivation in Universal Design for Learning within Costa Rica's Curriculum \\ Education Policy
}

Maybel Quirós Acuña

Ministerio de Educación Pública, Costa Rica

maycaqui@gmail.com

iD http://orcid.org/0000-0001-7923-187X

Mario Alberto Segura

Ministerio de Educación Pública, Costa Rica

mario.segura@ucr.ac.cr

iD http://orcid.org/0000-0003-1935-8021

DOI: https://doi.org/10.15517/revedu.v44i2.40360

Redalyc: http://www.redalyc.org/articulo.oa?id=44062184034

Recepción: 13 Febrero 2020

Aprobación: 05 Junio 2020

\section{Resumen:}

La política curricular: educar para una nueva ciudadanía (2015) debe tener la posibilidad de adquirir herramientas intelectuales, éticas y tecnológicas que le permitan desenvolverse de forma solvente en su vida, para lo cual se establece un aprendizaje integral y enfocado en el desarrollo de habilidades. Es congruente con lo planteado en el Diseño Universal para el Aprendizaje-DUACentro de Tecnología Especial Aplicada (CAST), (2018), el cual establece tres principios con base neurocientífica para poner el aprendizaje en todo el estudiantado. En este ensayo se da principal importancia al principio de múltiples formas de motivación, pues se parte de que el aprendizaje significativo solo se podrá lograr si la emoción y la motivación están presentes en el proceso de aprendizaje y de evaluación. Todo lo anterior, se concluye desde un enfoque sistémico del proceso de evaluación de los aprendizajes proporcionando tanto a estudiantes como a docentes la posibilidad de reconocer las señales que les brinda el contexto de estudio y que les indican lo que es un trabajo de buena calidad, para lo cual se destacan los procesos de autoevaluación y evaluación entre iguales, que a su vez permite el desarrollo de la habilidad de aprender a aprender o metacognición, la cual se cumple solo si el estudiantado puede planificar lo que va a aprender, regularse y evaluarse, convirtiéndose así en aprendiz experto.

Palabras ClaVe: Formas de implicación, Evaluación de los aprendizajes, Educación inclusiva, Política curricular.

\section{ABstract:}

Costa Rica's Curriculum Policy: Educating for New Citizenship (2015), must be flexible enough to be able to prepare students with the required intellectual, ethical and technological tools so that they will acquire life skills to make them more resilient. For this purpose, a comprehensive approach to learning is required that focuses on skills development. This is in sync with the Universal Design for Learning-UDL Guidelines- (Special Applied Technology Center (CAST), 2018), a framework which sets forth three neuroscientific principles to improve how students learn. This essay emphasizes the principle of multiple forms of motivation, based on the premise that meaningful learning can only be attained if emotion and motivation are present throughout the learning and assessment process. The aforementioned statements are the result of a systems-based approach to the learning and evaluation process, thereby providing both students and the teachers with insight for recognizing signs within the context of learning that represent good quality work. Both peer and self-assessment are also highlighted so that students can develop abilities for learning or metacognition. This is accomplished only when students are able to plan what they will learn as well as self-regulate and selfassess themselves and, ultimately, become an expert learner.

KEYWORDs: Forms of Engagement, Learning Assessment, Inclusive Education, Curriculum Education Policy. 


\section{Fundamentación filosófica de la política CURRicular, la PERSONA: CENTRo del PROCESO EDUCATIVO Y SUJETO TRANSFORMADOR DE LA SOCIEDAD}

La política curricular: educar para una nueva ciudadanía (2015), se fundamenta en cuatro paradigmas filosóficos, expuesto en la política educativa La persona: centro del proceso educativo y sujeto transformador de la sociedad, que son: la complejidad, el humanismo, el constructivismo social y el racionalismo. Derivado de lo anterior, la educación costarricense ha tomado la decisión de promover una transformación curricular. La cual se basa en los Derechos Humanos y Deberes de la Ciudadanía, que permita el desarrollo sostenible, con una visión planetaria con identidad nacional, con acceso a la tecnología con equidad social para que las personas puedan tomar decisiones y transformar sus contextos. Desde esta fundamentación se parte que más allá de que la población estudiantil deba aprender contenidos y repetirlos en pruebas escritas.

La educación ante todo debe ser promotora de la democracia como principio fundamental de colaboración en la comunidad educativa, en este espacio dialógico, surgen acuerdos, que permiten la convivencia entre las personas, siendo el diálogo la herramienta para el entendimiento en los disensos para la búsqueda de consensos.

En los procesos de enseñanza y aprendizaje, es necesario la búsqueda de un conocimiento que permita dialogar, interrelacionar, conectar, consensuar y participar en procesos de aprendizaje, esto es lo que se suele identificarse con el término de pedagogía.

Según Daniels (2003), citando a Bernstein (1999), la pedagogía es el proceso en el cual las personas adquieren conocimientos, conductas, prácticas y criterios de alguien o algo que se considere un facilitador y evaluador apropiado. Este adjetivo de apropiado, se concede porque quien aprender así lo considere o se da el aval por otros generalmente una organización o institución. Esta definición valida la importancia de los aspectos cognitivos y emocionales, en el proceso de aprendizaje.

Aunado a lo anterior, Ortiz (2016) menciona a Maturana (1996), que el lenguaje es un modo de estar en dominio de consenso, de coordinaciones de conducta, que requiere una plasticidad en ese espacio tan grande, que todos los seres humanos que crecen en el lenguaje son capaces de aprender cualquier cosa, mediante el diálogo en el saber pedagógico. Desde esta perspectiva, el lenguaje desarrolla pensamiento y este se construye en ambientes democráticos y participativos.

En consecuencia, el saber pedagógico debe venir acompañado de una práctica educativa humanista, centrada en los Derechos Humanos, que conlleve a una acción liberadora de los participantes, tal y como lo propone la Política Curricular. Por eso, es importante considerar a la persona aprendiente como sujeto y no como objeto o recipiente que debe ser llenado con conocimientos, sino como el constructor principal de los propios aprendizajes, adquiridos en su cotidianidad, adquiriendo su papel protagonista y de centro del proceso educativo.

Según Freire (1993), "para el educador progresista coherente, enseñar y aprender son momentos del proceso mayor de conocer. Por eso mismo implican búsqueda, viva curiosidad, equívoco, acierto, error, serenidad, rigor, sufrimiento, tenacidad, pero también satisfacción, placer y alegría”. (p.77). Esto se retoma en los cuatro paradigmas filosóficos plasmados en la Política Educativa "La persona: centro del proceso educativo y sujeto a transformación de la sociedad” (Ministerio de Educación de Pública de Costa Rica [MEP], 2017, p.1), los cuales son: el paradigma de la complejidad, el constructivismo social, el racionalismo y el humanismo, que se detallan a continuación.

El Paradigma de la complejidad: pretende promover que el ser humano salga del estado de desarticulación y fragmentación del saber contemporáneo, mediante la autorregulación, con la ayuda de los procesos metacognitivos: "El ser humano se caracteriza por tener autonomía e individualidad, establecer relaciones con el ambiente, poseer aptitudes para aprender, iniciativa, creatividad, capacidad de integrar información del mundo natural y social y la facultad de tomar decisiones" (MEP, 2017, p.11). 
El Humanismo es la base para la búsqueda de los ideales del ser humano a partir de la construcción del conocimiento; comprende la interacción de éste con los más altos valores inclusivos del desarrollo personal y social. El MEP (2017), toma en cuenta los valores inclusivos del humanismo. Estos valores se relacionan con la solidaridad y con la naturaleza, dignifican la esencia de los seres humanos; conllevan un concepto de humanidad integral en una relación de justicia con la diversidad cultural, biodiversidad biológica y están relacionados con la búsqueda de un estado de equilibrio a nivel fisiológico, emocional y mental.

Por otra parte, el constructivismo social, de acuerdo con la Política Educativa, pretende explicar la génesis, el origen del conocimiento y la forma en que la persona construye el conocimiento a partir de la interacción con el medio que le rodea tanto social como natural. "Esto se da en una interacción entre el nivel mental interno y el intercambio social. Es parte y producto de la actividad humana en el contexto social y cultural donde se desarrolla la persona" (MEP, 2017, p.12).

Por último, el racionalismo, “...que se sustenta en la razón y en las verdades objetivas como principios para el desarrollo del conocimiento válido, ha sido fundamental en la conceptualización de las políticas educativas costarricenses" (MEP, 2017, p.13), constituye el disfrute legítimo del ser humano, de la búsqueda del conocimiento que le permite el cultivo del intelecto; al mismo tiempo, crea un marco de referencia para crear y recrear su cultura,

Uno de los retos más grandes de cualquier política educativa es llevar a la práctica la fundamentación filosófica que propone, por lo que es en este aspecto que la Política Curricular (MEP, 2015), toma su relevancia, pues propone cuatro dimensiones y trece habilidades las cuales deben desarrollarse en todos los niveles y ofertas, tanto a nivel público como privado. Es aquí, donde se señala, la complementariedad de amabas políticas y como promueven llevar al acto educativo una transformación curricular que permita el desarrollo de las funciones ejecutivas de la población estudiantil.

Esta transformación curricular, propone la inclusión de la flexibilidad curricular, para responder a las diferentes características de la población estudiantil, mediante el Diseño Universal para el Aprendizaje (DUA), el cual constituye “...un marco que promueve la flexibilización del currículo al asumir la variabilidad individual como norma y no como excepción” (MEP, 2015, p.31).

El DUA, surge con los últimos avances neurocientíficos, proponiendo que el conocimiento se construye desde tres tipos de redes neuronales: afectivas, de planificación estratégica y de reconocimiento; cada una de estas redes se representa por medio de tres principios que establecen una ruta a seguir por el/la docente para diseñar sus clases desde una perspectiva de diseño universal, es decir para cada uno del estudiantado a su cargo.

Esta perspectiva, da como resultado una propuesta curricular flexible, que toma en cuenta las características individuales y plantea estrategias de medición pedagógica que permitan la participación y el aprendizaje de la población estudiantil.

El primer principio del DUA, establecido en la versión 2.2 del Centro Especial de Tecnología Aplicada (CAST), por sus siglas en inglés (CAST, 2018); debido a que en versiones anteriores aparecía como el tercer principio; considera que durante la mediación pedagógica es importante implicar y motivar al alumnado en el proceso de aprendizaje, para lo cual, según Lagos (2019), "considerar una planificación de clase que incorpore los intereses y preferencias de los estudiantes” (p. 259), es fundamental para la participación y el aprendizaje de todas las personas.

Por esta razón, en el presente artículo se focalizará en este principio, dejando los otros dos principios: múltiples formas de representación y múltiples formas que están articulados para disertaciones posteriores.

El principio de implicación y motivación del Diseño Universal para el Aprendizaje, como una vivencia del trabajo en el aula y en el centro educativo, se ha de considerar en el ambiente de aula, con el planteamiento de temas con relevancia social, como por ejemplo equidad de género, educación para la sexualidad, la prevención y atención de la violencia en y desde los centros educativos, estilos de vida saludable; ciudadanía digital, promoción de una cultura de paz; educación para la cultura ambiental para el desarrollo sostenible, entre muchos otros. 
En este mismo orden de ideas, "el DUA ayuda a tener en cuenta la variabilidad de los estudiantes al sugerir flexibilidad en los objetivos, métodos, materiales y evaluación que permiten a los educadores satisfacer diversas necesidades" (Álvarez y Moreno, 2015, p.12), lo que impacta directamente en la implicación del estudiantado en el proceso de mediación pedagógica vislumbrándolo como un aprendiz experto.

Esta experticia en el aprendizaje, se logra promoviendo la oportunidad de desarrollar la metacognición, es decir aprender a aprender. Que implica tres procesos, que según Ulate (2014), están implícitos en la capacidad metacognitiva los cuales son la planeación, el monitoreo o autorregulación durante el aprendizaje y la evaluación al terminarlo, logrando que la persona se evalúe y sea haga responsable de su propio proceso para aprender, modificándolo y perfeccionándolo día a día.

El propósito de este ensayo, consiste en brindar importancia al principio de múltiples formas de motivación, pues se parte de que el aprendizaje significativo solo se podrá logar si la emoción y la motivación están presentes en el proceso de aprendizaje y de evaluación.

\section{MÚLTIPLES FORMAS DE IMPLICACIÓN Y MOTIVACIÓN}

Las múltiples formas de implicación o motivación son fundamentales para que se construya el aprendizaje, para Capra (1996), "la inteligencia humana, la memoria humana, los juicios humanos nunca son completamente racionales sino que están teñidos por las emociones. Nunca podemos separar la racionalidad humana de la emoción, ni tampoco de la intuición" (p.29).

Es por esto que el aprendizaje cargado de emoción y motivación, logra que la población estudiantil mejore en sus procesos de atención y por ende se mostrará persistente, logrando así aprendizajes significativos que lleguen a transformar su realidad, convirtiéndose en sujeto transformador de la sociedad.

Aunado a lo anterior, el MEP (2017), asume la persistencia e implicación como punto nuclear que articula otros principios clave de la inclusión y equidad:

El respeto a la diversidad, la multiculturalidad y pluriculturalidad, la igualdad, de género, la sostenibilidad, la resiliencia y solidaridad, asi como las metas educativas que fomentan la formación humana para la vida, con el desarrollo de habilidades, destrezas, competencias, actitudes y valores (p.12).

El saber pedagógico es por sí mismo humanizante, con los principios éticos de quienes participan, donde priva la coherencia entre el ser y el hacer del personal docente. Por tanto, el centro educativo debe convertirse en un agente de cambio social, promoviendo la solución de los problemas de la comunidad, al mismo tiempo que los procesos de enseñanza y aprendizaje sean de calidad, en un ambiente motivante y retador, que permita constantemente la autorregulación y evaluación de lo que se hace.

Por tanto, se requiere un proceso de aprendizaje donde el estudiantado tenga el derecho a indagar, desaprender, reaprender, al error, al gusto, el riesgo y a la alegría de aprehender y de cuidar el mundo como propio. El error entonces se visualiza dentro de un ambiente de clase democrático, como una oportunidad para aprender y mejorar cada día y no como causa de vergüenza o de señalamiento por parte del/la docente.

Asimismo, es importante un personal docente comprometido éticamente con la democracia y convivencia de su práctica educativa, por lo que requiere una formación permanente científica, pedagógica y política; así como una actitud por aprender-aprender y renovarse en sus prácticas, reflexionando sobre su propio trabajo y mejorándolo de forma sistemática.

La comunidad también es partícipe de una práctica educativa comprometida, por lo que debe tener una plena participación en la formación de valores inclusivos, con el propósito de desarrollar el sentido de pertenencia que a su vez motiva a quienes participan.

Las formas de implicación y motivación en el salón de clases, proporcionan situaciones que favorezcan la autorregulación del estudiantado y las otras funciones ejecutivas del cerebro, por medio de tres pautas con sus puntos de verificación, a saber: 
- Captar el interés: permitiendo a cada estudiante elegir los temas, dando oportunidad para establecer diversas rutas para abordarlos y estableciendo ejercicios donde siempre hay probabilidad de éxito.

- Mantener el esfuerzo y la persistencia: dejando claro los objetivos y metas por alcanzar, con retos cognitivos que se resuelvan por medio de la comunicación y colaboración.

- Autorregulación: motivar cada logro, visualizar el error como oportunidad para aprender, desarrollando la autoevaluación y la reflexión.

Paso fundamental para este principio es que, durante la mediación pedagógica, se fomente la reflexión, el intercambio de opiniones y la argumentación como la comprensión de los puntos de vista del estudiantado, es decir, consensuar y difundir las normas de convivencia.

Lo anterior, permite en el alumnado, captar el interés, la persistencia y la autorregulación, con la promoción y la exposición de ideas, opiniones, experiencias. Lo cual conlleva al fomento de las manifestaciones cotidianas de diversidad cultural durante el proceso de mediación pedagógica, procesos de evaluación sobre manifestaciones y conflictos originados por la diversidad cultural y sobre las diferentes resoluciones, lo cual posibilita la comunicación y exposición de trabajos e investigaciones desarrolladas tanto a nivel curricular como cocurricular.

\section{LA FORMACIÓN EN VALORES COMO UNA FORMA DE IMPLICACIÓN}

La formación para la vivencia de los valores inclusivos, es un trabajo permanente en todo el quehacer educativo, sea en el centro educativo, en el hogar y en la comunidad y debe ser abordado a partir de las vivencias cotidianas, para lograr la práctica en los centros educativos, el personal docente planea estrategias metodológicas significativas, interesantes, motivadoras y creativas, sin que esto signifique trabajo adicional.

Es importante, partir de las experiencias previas, las expectativas, los intereses y necesidades de la población estudiantil. Asimismo, se busca propiciar la toma de conciencia en el estudiantado para la vivencia de los valores inclusivos de acuerdo con la realidad en que se hallan inmersos.

Se parte de la premisa que el estudiantado mediante la interacción respetuosa con sus familiares, educadores y demás personas que se encuentran a su alrededor, en las prácticas cotidianas, logre recrearse y solidificarse en los términos que son necesarios en el presente momento, con el fin de que en la mediación pedagógica que ocurre en cada clase, se logren sumar esfuerzos que impacten a su vez en cada centro educativo, región educativa y al final en el sistema mismo, en procura de una educación de calidad, la cual necesariamente debe ser inclusiva:

Habremos de analizar esta cuestión con cuidado, con el objeto de fomentar y no de entorpecer, las necesarias sinergias que desde distintos ámbitos, organizaciones y movimientos sociales se deben llevar a cabo para hacer frente a las barreras que limitan el derecho a una educación inclusiva, esto es, el derecho a la equiparación de oportunidades para conseguir una verdadera educación de calidad para todos, sin exclusiones ni eufemismos. (Echeita y Ainscow, 2011, p.30).

Según Lagos (2019), las actividades en que participen el estudiantado dentro y fuera del centro educativo deben estar impregnadas del sano ejercicio de los valores inclusivos:

... es el componente emocional, es decir, considerar una planificación de clase que incorpore los intereses y preferencias de los estudiantes. Las opciones, para ajustar las demandas y proporcionar apoyos en la práctica pedagógica, según este principio deben considerar opciones para captar el interés del estudiante, opciones para mantener el esfuerzo y la persistencia, y opciones para desarrollar la capacidad de autorregularse (p. 259).

Es fundamental, cambiar la concepción de mediación pedagógica, por una universalmente válida, donde todas las personas tengan deseos de aprender, amando lo que crean, por ejemplo, un(a) estudiante requiere de apoyos educativos en el proceso de lectura y escritura, una alternativa puede ser la lectura fácil, como “...un planteamiento general sobre la accesibilidad a la información y a la comprensión de los mensajes escritos de 
las personas con diversidades intelectuales y de aprendizaje" (Grupo de Educación y Diversidad, 2009 citado en García, 2012, p. 22), por lo que se recomienda el análisis de cuentos, ensayos, poesías, periódicos, revistas y otros documentos propiciando que sean entendibles por la mayoría de las personas eliminado barreras en la comunicación y la comprensión.

\section{El PAPEl De La EVALUACión De los APRENDIZAJES EN LAS FORMAS IMPLiCACión}

La evaluación de los aprendizajes, le permite a la población estudiantil, que constantemente esté aprendiendo mediante los procesos formativos, estos exigen que el cuerpo docente tenga la capacidad de representar múltiples maneras de aprendizaje para que el estudiantado llegue a las habilidades planteadas, mediante el papel fundamental de la autoevaluación y coevaluación, para que el mismo sea consciente sobre su propia desempeño e implemente diversas estrategias de demostrar lo aprendido.

La evaluación de los aprendizajes, coadyuva a superar barreras binarias respecto a conocimientos adquiridos o no, a valorar el desempeño de cada estudiante mediante diversas estrategias e instrumentos y a proveer información pertinente centrada en los procesos de enseñanza y aprendizaje a corto, mediano y largo plazo:

El feedback que fomenta la autorregulación es aquel que logra que el estudiante desarrolle mecanismos que lepermitan apropiarse de los objetivos, construir una base de orientación que haga posible anticipar y planificar la acción para alcanzar estos objetivos y conocer y aplicar los criterios de evaluación (Cano, 2016, p.11).

En el sistema de evaluación tradicional, no se prepara al estudiantado para tener éxito, es decir, nunca se le realimenta para mejorar su propio desempeño. Si el personal docente no cree en sus estudiantes, muy difícilmente se genere un ambiente positivo para ellos. "Las prácticas de evaluación deben contribuir a la construcción de la confianza de los estudiantes en su capacidad para aprender" (Boud. 2000, p. 6).

De la misma manera la evaluación formativa, brinda una realimentación con el fin de la mejora constante de la población estudiantil, llevar un registro con los avances que presenta el estudiantado, para que logre el proceso de la autorregulación, de tal forma que lleven un control de sus conocimientos; así como la importancia de promover en el estudiantado la capacidad de resolución de problemas, relacionados con la vida cotidiana, es decir, que sean relevantes en las propias vivencias.

Por consiguiente, la realimentación del/la docente parte de los propios errores del estudiantado, por medio de la dimensión de maneras de pensar, según la Política de Transformación Curricular (MEP, 2015), propicia las habilidades de: "El pensamiento sistémico, el pensamiento crítico, el aprender a aprender; la resolución de problemas y la creatividad e innovación" (p.29).

Lo anterior, se lograría cuando el/la estudiante tenga la capacidad de emitir juicios de valor o poseer criticidad, acerca de los problemas locales y nacionales que nos aquejan, proponiendo desde su creatividad e innovación formas diversas de resolverlos.

\section{REFLEXiOnes FinALES}

Los diferentes informes del Estado de la Educación han señalado la necesidad de mejorar la calidad de la educación. Esta demanda no solo de esta instancia sino de la sociedad costarricense en general exige que las políticas educativas realmente encaminen el accionar de todo el sistema hacia la consecución de esta meta.

Es por lo anterior, que un gran desafío de cualquier sistema educativo es llevar a la práctica las políticas, pues se perciben como un documento más y no como una ruta de trabajo. Por lo que, el esfuerzo que se ejecuta actualmente desde el Ministerio de Educación Pública, es un hito en la historia dado que pretende alcanzar niveles más altos de calidad promoviendo el cambio desde arriba hacia abajo, es decir desde el propio acto educativo. 
Para lograr lo anterior, se requiere de un gran compromiso de todos los niveles e instancias del Magisterio nacional, iniciando por un cambio de perspectiva promoviendo que cada estudiante sea el centro del proceso educativo, brindando múltiples formas para motivarlo e implicarlo en el proceso de aprender a aprender convirtiéndolo en un aprendiz experto

La organización de la clase es uno de los aspectos fundamentales que no requieren de mucha inversión pero que pueden impactar enormemente en el aprendizaje, por ejemplo: abandonar las filas tradicionales y permitirse el trabajo colaborativo y cooperativo, proyectos colectivos de investigación; aprendizaje basado en proyecto, que favorecen la promoción de habilidades sociales en el estudiantado, las cuales según Booth y Ainscow (2011), conllevan a poner en práctica establecer estructuras, procedimientos y actividades inclusivas: los valores inclusivos de igualdad, participación, comunidad, respeto a la diversidad y sostenibilidad.

Por otro lado, una de las situaciones que son frecuentes en la realidad educativa del país, es que se cree que la evaluación de los aprendizajes puede ser sostenible por su carácter instrumental, por lo que no se toma en cuenta que se presenta en la manera en la que la población estudiantil responde a las características de la tarea y en las formas que este se implique en su propio proceso de aprendizaje.

Por ejemplo, muchos docentes tienen la costumbre de pensar que si dejan un trabajo o proyecto de investigación ya están cumpliendo con la evaluación formativa, pero "una actividad sostenible en una situación o para un estudiante puede no serlo para otro. Una tarea de evaluación no puede ser juzgada como una contribución al aprendizaje sostenible simplemente a través de la inspección de las instrucciones dadas a los estudiantes" (Boud, 2000, p.12).

La evaluación de los aprendizajes en Costa Rica, cuando es entendida erróneamente, fomenta muy poco la autoevaluación y la reflexión, aspectos fundamentales de la metacognición y de la motivación, el/la docente es quien toma el papel del evaluador de forma unilateral. Esto no permite que el estudiantado aprenda a preguntarse por su proceso de aprendizaje, parece que se desconoce que la autoevaluación mejora, en gran medida, el rendimiento de los aprendientes.

Por otra parte, favorecer el proceso de formación en valores inclusivos, es precisamente ayudar al estudiantado a reconocer las señales que les brinda el contexto de estudio y que les indican lo que es un trabajo de buena calidad; además, desarrollar criterios que les permitan distinguir la buena, de la no tan buena ejecución de los trabajos.

Es decir, favorecer a la población estudiantil a reconocer indicios externos que pueden utilizar para dar realimentación a sí mismos. Es necesario, por lo tanto, incorporar en el pensamiento del estudiantado la importancia de la evaluación formativa, para que ellos sean capaces de aplicarla en cualquier proceso de aprendizaje a lo largo de su vida.

Asimismo,

descentrar el ego debe ser un requisito previo para una evaluación eficaz, esto debido a que se centra regularmente en lo que 'pueda ofrecer' el estudiantado y no en lo que se quiere lograr. De manera que el estudiante le dará más importancia a la nota y no al proceso de aprender (Boud, 2000, p.7).

\section{REFERENCIAS}

Álvarez, D y Moreno, M. (2015). Tecnologías de apoyo basadas en el diseño universal para el aprendizaje. Universidad de Colombia. Colombia: Graphic in S.A.S. Recuperado de https://www.researchgate.net/publication/337544805

Booth, T. y Ainscow. M. (2011). Index for Inclusion. Developing learning and participation in schools. United Kingdom: CSIE.

Boud, D. (2000). Sustainable assessment: rethinking assessment for the learning society. Studies in Continuing Education, 22(2), 151-167. Australia. doi, https://doi.org/10.1080/713695728 


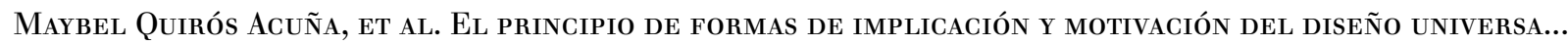

Cano, E. (2016). Del feedback al feedforward. En N. Cabrera y M.R. Mayordomo (Eds.), Feedforward formativo en la universidad (pp. 31-40) Barcelona, España: Transmedia XXI. Recuperado de https://www.redalyc.org/pdf/ 567/56729526017.pdf

Capra, F. (1999). El Punto Crucial. Buenos Aires: Editorial Estaciones.

Centro de Tecnología Especial Aplicada (CAST) (2018). Universal design for learning guidelines. Versión 2.2 (graphic organizar). Recuperado de https://www.thinglink.com/scene/1115219630927904771

Daniels, H. (2003). Vigotsky y la pedagogía. Buenos Aires: Ediciones Paidós.

Echeita, G. y Ainscow, M. (2011). La educación inclusiva como derecho. Marco de referencia y pautas de acción para el desarrollo de una revolución pendiente. Revista Tejuelo, (12), 26-46. Madrid. España.

Freire, P. (1993). Política y educación. Madrid: Siglo XXI.

García, O. (2012). Lectura fácil, métodos, de redacción y evaluación. España: Real Patronato sobre Discapacidad.

Lagos, O. (2019). Diseño universal para el aprendizaje: una experiencia innovadora en el aula matemática de octavo año básico. Revista de Estudios y Experiencias en Educación, 18(36), 257-267. doi, https//:doi.org/10.21703/ rexe.20191836lagos3

Maturana, H. (1996). El sentido de la humano. Santiago de Chile: Dolmen Ed.

Ministerio de Educación Pública (MEP). (2015).Fundamentación Pedagógica de la Transformación Curricular: Educar para una nueva ciudadanía. San José, Costa Rica: CSE.

Ministerio de Educación Pública (MEP). (2017). Política Educativa: La persona: Centro del proceso educativo y sujeto transformador de la sociedad. San José Costa Rica: CSE. Recuperado de https://www.mep.go.cr/politica-educ ativa

Ortiz, A. (2016). Humberto Maturana Nuevos Paradigmas en el Siglo XXI. Psicología, Educación y Ciencia. Chile: EDIBERUM.

Ulate, M. (2014). Mediación pedagógica que promueve el desarrollo de las funciones ejecutivas metacognitivas en los procesos de lectura y escritura que desarrollan los estudiantes de II ciclo de primaria de la dirección regional de educación de occidente. (Tesis de doctorado). Universidad de Costa Rica. San José, Costa Rica.

\section{BY-NC-ND}

